\title{
A Case of Severe COVID-19 Pneumonia Diagnosed in Bronchoscopy With Negative Repeated Nasopharyngeal Swabs
}

\author{
Yousef Shukhaa, b (i), Kamal Makhoula, b, Jawad Abu-Elhija ${ }^{\text {a, b }}$,

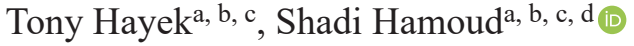

\begin{abstract}
Coronavirus disease 2019 (COVID-19) is a worldwide pandemic that had emerged in China since December 2019. The disease affects all age groups, with clinical manifestations in the spectrum from asymptomatic to rapidly lethal multi-organ failure, mainly involving the respiratory system. Diagnosis is confirmed mainly by a positive real-time polymerase chain reaction (PCR) nasopharyngeal swab. It is highly recommended to avoid performing invasive procedures in COVID-19 subjects to prevent the potential for dissemination of the pathogen. Treatment consists in particular of respiratory support and symptom relief. Dexamethasone is widely used with encouraging response. There were no cases in the literature that were diagnosed with positive reverse transcription-polymerase chain reaction (RT-PCR) testing only from fluid of involved organs, while repeated nasopharyngeal swabs returned negative for COVID-19. We here describe a case of COVID-19 that presented with moderate-severe pulmonary involvement, diagnosed by RT-PCR testing from bronchoalveolar lavage, while several nasopharyngeal swabs were consistently negative. The patient experienced no improvement under widespectrum antibiotics administered initially, and greatly improved after receiving systemic corticosteroids. One can realize from our case that COVID-19 could not be ruled out upon repeated negative RT-PCR nasopharyngeal swabs, and in subjects with highly suspected COVID-19, it is justified to perform invasive procedures, but still using maximal protective measures.
\end{abstract}

Keywords: COVID-19; Nasopharyngeal swab; RT-PCR; bronchoscopy

Manuscript submitted February 8, 2021, accepted February 22, 2021

Published online March 24, 2021

a Department of Keter B, Rabmbam Health Care Campus, Haifa, Israel bepartment of Internal Medicine E, Rabmbam Health Care Campus, Haifa, Israel

${ }^{\mathrm{C}}$ Rappaort Faculty of Medicine, Technion, Israel Institute of Technology, Haifa, Israel

${ }^{\mathrm{d}}$ Corresponding Author: Shadi Hamoud, Department of Keter B, Rabmbam Health Care Campus, Haifa, Israel. Email: s_hamoud@rmc.gov.il

doi: https://doi.org/10.14740/jmc3679

\section{Introduction}

Coronavirus disease 2019 (COVID-19) is a pandemic that was first reported in a cluster of patients in Wuhan city, China, in December 2019. The natural history of the disease is flulike, typically presents with high-grade fever, cough, throat pain, loss of smell and taste, headache and myalgia. Common laboratory findings include lymphopenia, elevated C-reactive protein and D-dimer levels, the last representing diffuse endothelial injury. Elevated liver enzymes and renal function impairment occur frequently. Diffuse patchy lung infiltrates are the characteristic radiographic findings on chest radiograph or computed tomography (CT) scans. Diagnosis is confirmed by real-time polymerase chain reaction (PCR) (reverse transcription-polymerase chain reaction (RT-PCR)) testing from nasopharyngeal swabs for the coronavirus.

Treatment is mainly supportive and includes oxygen, respiratory support and anti-inflammatory systemic corticosteroids. Anti-coagulants are used to prevent or treat thromboembolic complications that commonly occur in COVID-19 patients, apparently due to the endothelial injury. The use of anti-viral and immunologic preparations is controversial. Several anti-viral vaccines are under development, and some are in world-wide clinical use.

\section{Case Report}

A 63-year-old female was admitted to an internal medicine department in our tertiary university medical center, with a history of 1-week high-grade fever, productive white colored cough, dyspnea and generalized myalgia. Her symptoms started 2 days after receiving the first dose of BNT162B2 Pfizer messenger ribonucleic acid (mRNA) vaccine for COVID-19. She denied exposure to documented COVID-19 patients.

Her past medical history is remarkable for hypertension treated with bisoprolol and ramipril, multiple sclerosis treated with fampridine and ocrelizumab, and hypercholesterolemia treated with atorvastatin.

On physical examination, the patient looked ill, with peak temperature of $39.4{ }^{\circ} \mathrm{C}$, blood pressure of $150 / 98 \mathrm{~mm} \mathrm{Hg}$, heart rate of $87 \mathrm{bpm}$ and room air $\mathrm{O}_{2}$ saturation of $95 \%$. Lungs were clear on auscultation and the rest of the physical exami- 


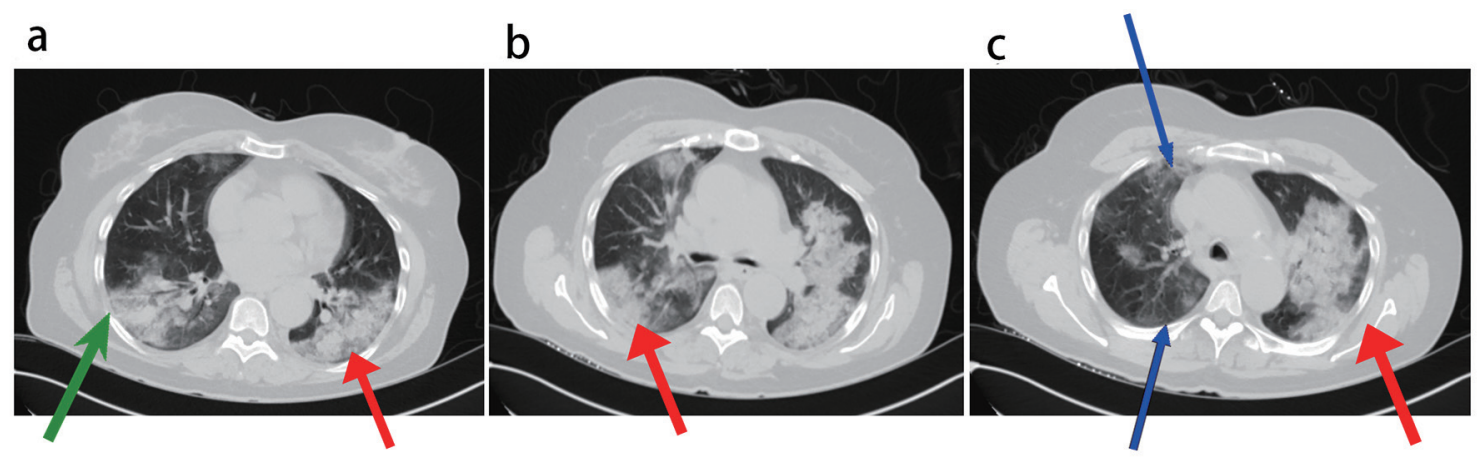

Figure 1. Thoracic tomographic scan images showing diffuse bilateral lung infiltrates (a-c, red arrows), an infiltrate with air bronchogram (a, green arrow), and infiltrates with ground glass opacities (c, blue arrow).

nation was unremarkable.

Blood tests revealed normal complete blood count and kidney function. C-reactive protein was elevated to $5 \mathrm{mg} / \mathrm{dL}$ (normal range $0-0.5 \mathrm{mg} / \mathrm{dL}$ ). Chest X-ray revealed patchy lung infiltrates, mainly in the right upper lobe. Nasopharyngeal swab test for COVID-19 was negative.

The patient was diagnosed with community acquired pneumonia and was treated with levofloxacin and azithromycin. During the first 5 days, the patient continued with high-grade fever, dyspnea and productive cough, while four repeated nasopharyngeal swabs for COVID-19 were negative. Thoracic CT scan revealed multiple bilateral pulmonary infiltrates, part of which are with ground glass opacity, and others with airbronchogram, raising the main differential diagnosis of infectious process versus a pulmonary hemorrhage (Fig. 1). Fiberoptic bronchoscopy was performed, in which bronchomalacia and pus-like discharge were noted in the airways of both lungs. Bronchial lavage testing was positive for COVID-19 RT-PCR. Following bronchoscopy, the patient experienced worsening dyspnea with decreased $\mathrm{O}_{2}$ saturation down to $92 \%$ on room air, necessitating oxygen supplementation through a nasal cannula. After bronchoscopy, repeated nasopharyngeal swabs for COVID-19 RT-PCR were still negative despite positive PCR for COVID-19 in the bronchoalveolar lavage.

The patient was transferred to the Keter Department (into which only patients with documented COVID-19 were admitted), and treated with intravenous dexamethasone and subcutaneous enoxaparin (for thromboembolism prevention), as well as oxygen supplementation of $3 \mathrm{~L} / \mathrm{min}$ using a nasal cannula.

Prominent improvement was noted. The high-grade fever and dyspnea resolved and cough was relieved. The patient was discharged on the seventh day in a good condition with recommendation to complete a 10-day protocol of dexamethasone treatment.

\section{Discussion}

The National Library of Medicine (Medline) search showed no studies regarding diagnosis of COVID-19 in visceral organs in the presence of negative nasopharyngeal swabs. In the letter to the editor by $\mathrm{Su}$ et al, the authors recommended to avoid performing bronchoscopy in subjects with symptoms suspected to be secondary to COVID-19, because of increased risk for dissemination of the virus [1]. Similarly, the US Centers for Disease Control and Prevention recommended to perform bronchoscopy only when necessary, with extreme caution by using N95 facial mask, goggles and a protective gown, as well as using the positive pressure respiratory hood when possible [2]. In an additional letter to the editor, Hao et al reported a case of a COVID-19 patient who presented with symptoms suggestive for COVID-19, together with lung infiltrates typical for COVID-19 involvement. In their case, the initial three consecutive nasopharyngeal swabs were negative for COVID-19 RT-PCR, and only the fourth test was positive [3]. Lastly, in a review of five different cases of symptomatic undiagnosed COVID-19 patients who presented with respiratory complaints but with negative RT-PCR nasopharyngeal swabs, early chest CT scans managed to reveal characteristic COVID-19 lung findings prior to diagnosis, and helped in isolating those patients to perform recurrent RT-PCR nasopharyngeal swabs until final diagnosis [4].

\section{Conclusions}

We are reporting a case of COVID-19 in a 63-year-old female, with negative repeated nasopharyngeal swabs for coronavirus RT-PCR, in spite of a simultaneous positive swab taken from a bronchial lavage.

To the best of our knowledge, this is the first case in literature of COVID-19 with severe pulmonary involvement that was diagnosed only though a visceral organ positive RT-PCR test, despite parallel several negative recurrent nasopharyngeal swabs. Prior to the definite COVID-19 diagnosis, the patient received wide spectrum antibiotics for suspected community acquired pneumonia, with no effect on the disease course, and only after corticosteroid initiation, the disease had completely resolved.

This case emphasizes the fact that a negative nasopharyngeal COVID-19 swab result does not rule out the possibility of active COVID-19 infection, even when repeated swabs are negative, and especially in patients with clinical, laboratory, and radiologic findings highly suggestive for COVID-19 infection.

Hence, among patients with high suspicion for COVID-19 infection but with negative nasopharyngeal swabs, we do recommend performing invasive tests from involved organs, 
while applying the maximal protective measures for the staff, in order to validate the diagnosis of the disease.

\section{Acknowledgments}

None to declare.

\section{Financial Disclosure}

None to declare.

\section{Conflict of Interest}

None to declare.

\section{Informed Consent}

Informed consent was obtained.

\section{Author Contributions}

YS, KM, JAH and SH were involved in daily follow-up and treatment of the patient. TH and SH were involved in daily management of the patient. SH wrote and edited the final version, and all authors reviewed the manuscript prior to submission.

\section{Data Availability}

Data regarding the patient will be available in the journal website.

\section{References}

1. Su ZQ, Ye TS, Chen DF, Deng XL, Chen HJ, Li SY. The role of bronchoscopy in COVID-19. Respiration. 2020;99(8):697-698.

2. Siegel JD, Rhinehart E, Jackson M, Chiarello L, Health Care Infection Control Practices Advisory C. 2007 guideline for isolation precautions: preventing transmission of infectious agents in health care settings. Am J Infect Control. 2007;35(10 Suppl 2):S65-164.

3. Hao W, Li M. Clinical features of atypical 2019 novel coronavirus pneumonia with an initially negative RTPCR assay. J Infect. 2020;80(6):671-693.

4. Xie X, Zhong Z, Zhao W, Zheng C, Wang F, Liu J. Chest CT for typical coronavirus disease 2019 (COVID-19) pneumonia: relationship to negative RT-PCR testing. Radiology. 2020;296(2):E41-E45. 